\title{
Pathogenicity Levels of Colombian Strains of Candida auris and Brazilian Strains of Candida haemulonii Species Complex in Both Murine and Galleria mellonella Experimental Models
}

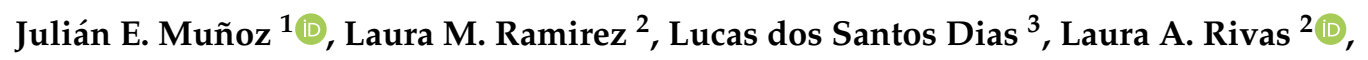 \\ Lívia S. Ramos ${ }^{4}$, André L. S. Santos ${ }^{4}$ (D) Carlos P. Taborda ${ }^{3,5}$ and Claudia M. Parra-Giraldo ${ }^{2, *(D)}$ \\ 1 MICROS Group, Medicine Traslacional Institute, School of Medicine and Health Sciences, \\ Universidad del Rosario, Bogotá, D.C. 111221, Colombia; juliane.munoz@urosario.edu.co \\ 2 Unidad de Proteómica y Micosis Humanas, Grupo de Enfermedades Infecciosas, \\ Departamento de Microbiología, Facultad de Ciencias, Pontificia Universidad Javeriana, \\ Bogotá, D.C. 110231, Colombia; lramirez-r@javeriana.edu.co (L.M.R.); lrivas@usp.br (L.A.R.) \\ 3 Department of Microbiology, Biomedical Sciences Institute, University of São Paulo (USP), \\ São Paulo, SP 05508-060, Brazil; dossantosdia@wisc.edu (L.d.S.D.); taborda@usp.br (C.P.T.) \\ 4 Laboratório de Estudos Avançados de Microrganismos Emergentes e Resistentes, \\ Departamento de Microbiologia Geral, Instituto de Microbiologia Paulo de Góes (IMPG), \\ Centro de Ciências da Saúde (CCS), Universidade Federal do Rio de Janeiro (UFRJ), \\ Rio de Janeiro 21941-901, Brazil; liviaramos2@yahoo.com.br (L.S.R.); andre@micro.ufrj.br (A.L.S.S.) \\ 5 Laboratory of Medical Mycology-LIM53/IMTSP, University of São Paulo (USP), \\ São Paulo, SP 05508-060, Brazil \\ * Correspondence: claudia.parra@javeriana.edu.co; Tel.: +57-1-3208320 (ext. 4305)
}

Received: 9 June 2020; Accepted: 5 July 2020; Published: 10 July 2020

\begin{abstract}
Candida auris and Candida haemulonii complex (C. haemulonii, C. haemulonii var. vulnera and C. duobushaemulonii) are phylogenetically related species that share some physiological features and habits. In the present study, we compared the virulence of these yeast species using two different experimental models: (i) Galleria mellonella larvae to evaluate the survival rate, fungal burden, histopathology and phagocytosis index and (ii) BALB/c mice to evaluate the survival. In addition, the fungal capacity to form biofilm over an inert surface was analyzed. Our results showed that in both experimental models, the animal survival rate was lower when infected with $C$. auris strains than the C. haemulonii species complex. The hemocytes of G. mellonella showed a significantly reduced ability to phagocytize the most virulent strains forming the C. haemulonii species complex. Interestingly, for $C$. auris, it was impossible to measure the phagocytosis index due to a general lysis of the hemocytes. Moreover, it was observed a greater capability of biofilm formation by C. auris compared to $C$. haemulonii species complex. In conclusion, we observed that $C$. auris and C. haemulonii complex have different levels of pathogenicity in the experimental models employed in the present study.
\end{abstract}

Keywords: Candida auris; Candida haemulonii species complex; virulence; candidiasis; biofilm; Galleria mellonella

\section{Introduction}

Candida auris and Candida haemulonii complex (C. haemulonii, C. haemulonii var. vulnera and C. duobushaemulonii) are phylogenetic related species, being the first one constantly related with healthcare-related infectious diseases (HID). These yeast species present ubiquitously distribution and 
inherently multidrug-resistance profile, especially against polyenes (e.g., amphotericin B) and azoles (e.g., fluconazole, itraconazole and voriconazole) [1].

Candida auris was isolated for the first time from the external auditory canal of a female patient in Japan in 2009 and in 15 South Korean patients in the same year [2,3]. It is a multidrug-resistant agent mainly related with HID, showing high rate of mortality and diversity of susceptibility profiles. This pathogenic yeast was reported in countries as Venezuela (2012), India (2013), South Africa and Kuwait (2014), Colombia (2015), Spain (2016), United States of America (2016), Panama (2017), United Kingdom (2017) and it continues to expand around the world [4-11].

Among its mainly features, $C$. auris is able to colonize a variety of surfaces, from inert materials to multiple body parts, presenting a large range of clinical manifestations [12,13]; (C) auris infection treatment can be tough due to the high resistance index that presents to most antifungals. One of the best therapeutic options to control a C. auris infection is the use of echinocandins, however, cases of resistance to this antifungal have already been reported [13,14]; (C) auris also stands out for its versatility to adapt to saline environments and, therefore, to resist desiccation. Recently, it was described that the hospital environment is an important risk factor in the process of $C$. auris transmission, since in many cases the intrahospital disinfection process is not enough to eradicate this fungus from the surfaces, which facilitates the transmission to other patients [15]. These patients are generally those with serious underlying medical conditions, including hematological malignancies and other conditions resulting in immunosuppression $[6,13,16]$. Therefore, it is urgent and necessary to design efficient antifungal strategies that help to prevent possible deaths caused by C. auris infection [17].

Candida haemulonii was found for the first time inside the intestine of a blue-striped fish (Haemulon scirus) in 1962. The first description of human infection was reported in 1984 from the blood of a patient, who died of renal failure despite of therapy with amphotericin $B$ and flucytosine [18]. This yeast can present different clinical manifestations from onychomycosis to a deep mycosis in neonates in intensive care unit [19]. In fact, C. haemulonii is a complex formed by three genotypic differentiated species (C. haemulonii, C. haemulonii var. vulnera and C. duobushaemulonii). Candidiasis by C. haemulonii is rare compared to other species as $C$. albicans. However, it is important to note that these cases of candidiasis are severe due to the (multi) drug-resistance profile. Actually, resistance to fluconazole and amphotericin $B$ has been defined, as well as strains presenting resistant to echinocandins were also described $[14,20]$. However, different susceptibility assays have demonstrated that $C$. haemulonii can be vulnerable to micafungin and caspofungin [21].

Since 2006, a close phylogenetic relationship had been observed between strains of $C$. haemulonii complex and C. auris that could only be resolved up to 2009 with the work of Satoh and collaborators [3] and later ratified by Cendejas-Bueno et al. 2012 [22], where they could differentiate the C. haemulonii complex from $C$. auris. The aim of the present work is to compare the virulence of $C$. auris versus the three species forming the C. haemulonii complex by means of in vitro and in vivo assays using Galleria mellonella larvae and BALB/c mice as models.

\section{Materials and Methods}

\subsection{Microorganisms}

Five strains of Candida auris (Ca432, Ca446, Ca386, Ca885 and Ca881) isolated from 2 different regions of Colombia and belonged to the Latin American clade IV, were selected to the present study. The clinical isolates Ca432, Ca446 and Ca386 of C. auris were obtained from Proteomic and Human Mycosis Investigation Unit, of Universidad Javeriana. Ca885 and Ca881 strains were obtained from Centro de Investigaciones Microbiológicas del Cesar (CIMCE). Six strains of the C. haemulonii complex distributed as C. duobushaemulonii (LIPCh1, LIPCh6) C. haemulonii (LIPCh3, LIPCh7, LIPCh12) and C. haemulonii var. vulnera (LIPCh11) [14] were obtained from Laboratório de Estudos Avançados de Microrganismos Emergentes e Resistentes of Universidade Federal do Rio de Janeiro (UFRJ). Strain identification was carried out by molecular and MALDI-TOF tests to verify the received material, 
as it is shown in Table 1. Standard strains of C. albicans (SC5314 and ATCC10231) were also included as controls. All strains were maintained at $-80^{\circ} \mathrm{C}$. To perform the experiments, the fungi were subcultured in Sabouraud dextrose broth (Becton, Dickinson and Company; Sparks, NV, USA) at $37^{\circ} \mathrm{C}$ for $24 \mathrm{~h}$ at $150 \mathrm{rpm}$ before each assay. Entirely yeast panel utilized in this study were maintained and cultivate under the same conditions. However, the yeasts growth rates were different; $C$. auris duplicated after $2 \mathrm{~h}$, while yeasts of the $C$. haemulonii complex duplicated after $3 \mathrm{~h}$. To prepare the inoculum, the fungal cells were centrifuged and resuspended in a saline solution and, subsequently, quantified using a hemocytometer.

Table 1. Identification and antifungal susceptibility profiles of the C. haemulonii complex and C. auris strains included in this study.

\begin{tabular}{|c|c|c|c|c|c|}
\hline \multirow{2}{*}{ Strain } & \multirow{2}{*}{ Isolation Date } & \multirow{2}{*}{ Source } & \multirow{2}{*}{ Country } & \multicolumn{2}{|c|}{ Susceptibility Profile } \\
\hline & & & & AMB & FLZ \\
\hline C. auris 432 & 2014 & $\begin{array}{l}\text { Craniotomy } \\
\text { (Secretion) }\end{array}$ & Colombia & $\mathrm{R}$ & $\mathrm{R}$ \\
\hline C. auris 446 & 2015 & Blood culture & Colombia & $S$ & $\mathrm{R}$ \\
\hline C. auris 386 & 2015 & $\begin{array}{l}\text { Bone Tissue } \\
\text { (Biopsy) }\end{array}$ & Colombia & S & $\mathrm{R}$ \\
\hline C. auris 885 & 2016 & Blood culture & Colombia & $S$ & $\mathrm{R}$ \\
\hline C. auris 881 & 2016 & Cerebrospinal fluid & Colombia & $S$ & $\mathrm{R}$ \\
\hline $\begin{array}{l}\text { C. duobushaemulonii } \\
\text { LIPCh1 }\end{array}$ & 2005 & Finger nail & Brazil & $\mathrm{R}$ & $\mathrm{R}$ \\
\hline $\begin{array}{l}\text { C. duobushaemulonii } \\
\text { LIPCh6 }\end{array}$ & 2009 & Toe nail & Brazil & $\mathrm{R}$ & $\mathrm{R}$ \\
\hline C. haemulonii LIPCh3 & 2009 & Toe nail & Brazil & $\mathrm{R}$ & $\mathrm{R}$ \\
\hline C. haemulonii LIPCh7 & 2009 & Toe nail & Brazil & $\mathrm{R}$ & $\mathrm{R}$ \\
\hline C. haemulonii LIPCh12 & 2013 & Blood & Brazil & $\mathrm{R}$ & $\mathrm{R}$ \\
\hline $\begin{array}{l}\text { C. haemulonii var. vulnera } \\
\text { LIPCh11 }\end{array}$ & 2013 & Blood & Brazil & $\mathrm{R}$ & $\mathrm{R}$ \\
\hline
\end{tabular}

\subsection{Galleria mellonella}

The larvae were obtained from Scientia S.A. (Cali, Colombia) and maintained at $4{ }^{\circ} \mathrm{C}$ in dark conditions until the inoculation day. Before infection, decontamination of larvae was carried out using two different beakers, one with $250 \mathrm{~mL}$ of sterile distilled water and another with $250 \mathrm{~mL}$ of $0.1 \%$ sodium hypochlorite, in constant agitation for $30 \mathrm{~s}$. Groups with 10 larvae each were placed in petri plates at $37^{\circ} \mathrm{C}$ constantly to continue with the infection procedure.

\subsection{Galleria mellonella Infection}

The larvae were infected with $10 \mu \mathrm{L}$ of the inoculum $\left(5 \times 10^{4}\right.$ cells/larva) using insulin syringe in the proleg. After being inoculated, the larvae were placed at $37^{\circ} \mathrm{C}$ in appropriate containers for their maintenance. Each group was composed with 10 larvae, with a proximally weight between 275-330 mg.

\subsection{Survival in Galleria mellonella}

Twelve groups with 10 larvae each were selected and then infected with C. auris, C. haemulonii species complex or C. albicans SC5314 (control group). Death of the larvae was scored daily for 10 days. Results were analyzed statistically. 


\subsection{Fungal Burden in Galleria mellonella}

Different groups of infected larvae were sacrificed with $2 \mathrm{~h}$ or 3 days after infection. The whole larvae were macerated with tips in microtubes with $1 \mathrm{~mL}$ of $0.9 \% \mathrm{NaCl}$. After that, serial dilutions $(1 / 10,1 / 100$ and $1 / 1000)$ were made, and $100 \mu \mathrm{L}$ of each dilution were seeded on Sabouraud agar. The plates were incubated at $37{ }^{\circ} \mathrm{C}$ for $48 \mathrm{~h}$, and colony forming units (CFU) were counted. Each experiment was performed in triplicate.

\subsection{Histopathology of Galleria mellonella}

Infected larvae were sacrificed after $3 \mathrm{~h}$ in the case of $C$. auris or after 3 days in the case of C. haemulonii species complex. Immediately, after slaughter the larvae were fixed in $10 \%$ buffered formalin and embedded in paraffin for sectioning. Slices were stained with periodic acid-Schiff (PAS) and examined microscopically at 10× magnification (Optiphot-2; Nikon, Tokyo, Japan).

\subsection{Yeast Phagocytic Capacity of Galleria Mellonella hemocytes}

For this test, the larvae infected with C. auris were kept alive for $3 \mathrm{~h}$ and in the case of $C$. haemulonii were kept for 3 days. Subsequently, a small incision was made in the pseudo-leg to obtain a drop of hemolymph, which was smeared on glass slides. After drying at room temperature, Wright dye was used to stain the samples and then they were observed under a microscope with a $100 \times$ magnification. In each slide 100 hemocytes were counted, then the hemocytes that had yeast inside and those that were empty were also counted with the aim to obtain the phagocytic percentage.

\subsection{BALB/c Mice}

Thirty-six isogenic females of BALB/c mice (6-8 weeks old) were bred at the University of São Paulo (São Paulo, Brazil) in an animal facility under specific pathogen-free conditions, constant temperature, light and dark cycles 12/12 h, ad libitum feeding and under the supervision of a single person trained and specialized in laboratory animal care. Procedures involving animals and their care were conducted according to the local ethics committee and international rules. All experiments were approved by the Institutional Animal Care and Use Committee of Institute of Biomedical Sciences (ICB), in the University of São Paulo (042-127-02, 12 April 2012).

\subsection{Survival in $B A L B / c$ Mice}

Six female BALB/c mice per group were immunosuppressed intraperitoneally with 2 doses of cyclophosphamide; $96 \mathrm{~h}$ and $24 \mathrm{~h}$ before intravenous infection with $1 \times 10^{4}$ yeasts of $C$. auris (Ca432, Ca446), C. haemulonii (Ch3, Ch11) or C. albicans (ATCC 10231) as positive control. Since these strains of $C$. auris and $C$. haemulonii complex were the most virulent in the G. mellonella survival test, they were selected to carry out the present assay. The negative control group received phosphate-buffered saline (PBS). Three days after infection, another dose of cyclophosphamide was applied, and the treatment was maintained every 4 days to ensure the animal immunosuppression [23]. The mice survival was evaluated during 30 days post-infection. Animals that survive were immediately anaesthetized with $80 \mathrm{mg} / \mathrm{kg}$ ketamine and $10 \mathrm{mg} / \mathrm{kg}$ of xylazine (both from União Química Farmacêutica, Brazil) and then euthanized by cervical dislocation. Thus, the total experimental model duration was 34 days.

When the survival assay was carried out, rigorous monitoring of infected animals was done twice per day in order to detect any signs of advanced disease, pain or distress. Twenty six mice presented some of these symptoms and they were immediately euthanized to avoid any type of suffering, following the guidelines of the Guide for the Care and use of Laboratory animals. Ten animals died during the night with a rapid evolution of the disease, in this case, the mice were removed early the next day in order to avoid the stress of the other animals. 


\subsection{Biofilm Formation}

The capacity to form biofilm by the yeasts was determined according to the protocol of Pierce and collaborators [24]. The yeasts of C. auris (Ca386, Ca432, Ca446, Ca881 and Ca885), C. haemulonii (LIPCh1, LIPCh11 and LIPCh3) and C. albicans (SC5314) as a control, were incubated overnight in Sabouraud dextrose broth (Becton, Dickinson and Company; Sparks, NV, USA) at 150 rpm and $37^{\circ} \mathrm{C}$. After this time, the medium was centrifuged, washed twice with PBS, and the final pellet was resuspended in RPMI medium. The suspensions of $C$. haemulonii, $C$. auris and the positive group were adjusted a final density of $1 \times 10^{6}$ cells $/ \mathrm{mL}$. Then, $100 \mu \mathrm{l}$ of these suspensions was spread in 96-well polystyrene plates, with a minimum of 6 replicates for each fungus. The negative control was only RPMI medium. After incubation at $37^{\circ} \mathrm{C}$ for $24 \mathrm{~h}$, the medium was aspirated carefully (to avoid the biofilm disruption) and washed three times with PBS to remove planktonic cells. Once the wells were dry, a solution of 2,3-bis(2-metoxi-4-nitro-5sulfo-fenil)-2H-tetrazolium-5-carboxanilida (XTT, Sigma-Aldrich, Saint Louis, MO, USA) containing menadione was added and incubated for $2 \mathrm{~h}$. Finally, $75 \mu \mathrm{l}$ of the supernatants were transferred into a new plate and the absorbance at $490 \mathrm{~nm}$ was measured in spectrophotometry.

\subsection{Statistical Analysis}

Statistics were performed using GraphPad Prism version 6.0 (GraphPad Software, San Diego, CA, USA). Statistical comparisons were made by analysis of variance (one-way ANOVA) followed by a Tukey-Kramer post hoc test. $p$-values of $<0.05$ indicated statistical significance. In the case of the survival curve, the Log-rank (Mantel-Cox) test with $p$-values of $\leq 0.001$ was used to indicate statistical significance. A 95\% confidence interval was determined in all experiments.

\section{Results}

\subsection{Galleria mellonella Infected with C. auris Survive Less than the Larvae Infected with the C. haemulonii Complex.}

G. mellonella larvae showed a different survival profile depending on the fungal strain with they were infected (Figure 1A). C. auris strains Ca432, Ca386 and Ca446 together with C. albicans SC5314, used as a control strain, showed a significantly higher virulence than the remaining tested strains (Figure 1B-Group 1). The C. auris strains that showed lowest virulence were Ca881 and Ca885 (Figure 1B-Group 2). It is important to note that the two less virulent strains of C. auris were more lethal than the strains with greater virulence of $C$. haemulonii species complex. Regarding the strains belonging to the C. haemulonii complex, the most pathogenic ones were LIPCh3 and LIPCh7 (C. haemulonii) as well as LIPCh11 (C. haemulonii var. vulnera) (Figure 1B-Group 3), while the strains with the lowest virulence capability were LIPCh1 and LIPCh6 belonging to C. duobushaemulonii and LIPCh12 belonging to C. haemulonii (Figure 1B-Group 4). The majority strains forming the C. haemulonii complex showed low virulence in relation to the $C$. auris strains with an approximate larvae survival of $30 \%$ and in some cases $80 \%$ of survival.

\subsection{Galleria mellonella Infected with C. auris Displayed a High Fungal Burden}

The number of CFU considering the larvae infected with different strains of Candida species showed a significant interspecific difference. In some cases, the CFU from larvae infected with C. auris was similar or even higher than the control group (C. albicans SC5314) as is the case of the larvae infected with Ca881 that presented the highest number of CFU compared with the other strains of $C$. auris and C. albicans (Figure 2A). It is important to note that there was a difference between the fungal burden of larvae infected with the different species of the C. haemulonii complex. Comparing the pathogenicity of the three species that integrate the $C$. haemulonii complex, the larvae infected with C. haemulonii (LIPCh7) presented the highest fungal burden compared with the others infected with C. duobushaemulonii (LIPCh1 and LIPCh6), C. haemulonii. var. vulnera (LIPCh11) and C. haemulonii (LIPCh3) that presented an intermediate fungal burden (Figure 2B). Within the C. haemulonii complex, 
the species that presented the lowest fungal burden was C. haemulonii (LIPCh12). The fungal burden of larvae infected with the species forming the $C$. haemulonii complex was significantly lower compared to larvae that were infected with the control strain of $C$. albicans and the five strains of $C$. auris (Figure 2C).

\subsection{Larvae Infected with C. auris Leads to a Severe Tissue Invasion and Absence of Granuloma-Like Cell Aggregates}

Larvae infected with the C. auris (Ca432 and Ca386) strain showed a greater number of dispersed yeasts in the tissue, revealing a severe infection and invasion profile. At the same time, the formation of cellular aggregates was not observed. It is important to note that these two strains (Ca432 and Ca386) of $C$. auris did not form hyphae or pseudohyphae as it can be observed in the other strains of $C$. auris (Figure 3C-F). Contrarily, larvae infected with the most virulent species of the $C$. haemulonii complex (C. haemulonii (LIPCh3 and LIPCh7) and C. haemulonii var. vulnera (LIPCh11) with 3 days of infection showed a more controlled infiltration and well-defined formation of granuloma-like cell aggregates (black arrows) that could control the infection process (Figure 3A,B).

A

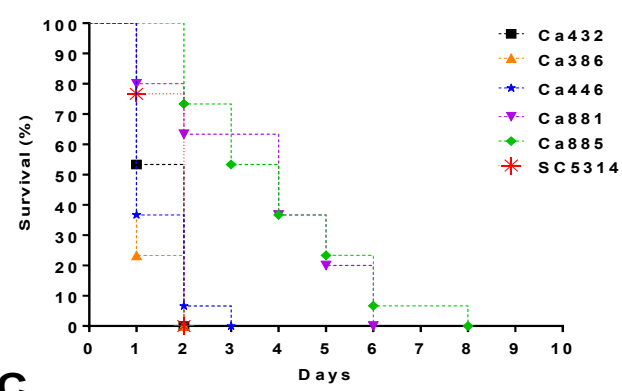

B

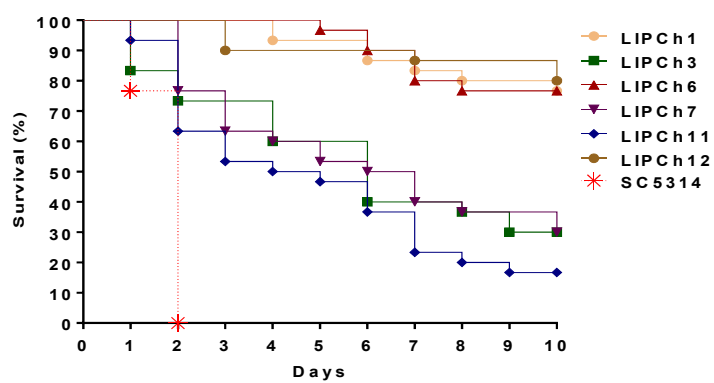

Pair comparison of survival curves by Long-rank (Mantel-Cox) test

\begin{tabular}{|c|c|c|c|c|c|c|c|c|c|c|c|}
\hline & Ch3 & Ch6 & Ch7 & Ch11 & Ch12 & Ca386 & Ca432 & Ca446 & Ca881 & Ca885 & SC5314 \\
\hline Ch1 & **** & NS & $\star \star \star *$ & **** & NS & 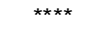 & 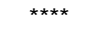 & 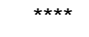 & 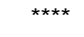 & 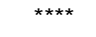 & 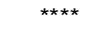 \\
\hline Ch3 & ND & 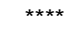 & NS & NS & 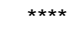 & 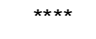 & 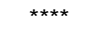 & 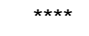 & 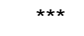 & ** & 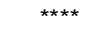 \\
\hline Ch6 & & ND & 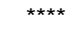 & 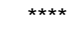 & NS & 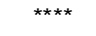 & 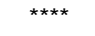 & 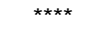 & $* * * *$ & 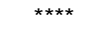 & 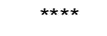 \\
\hline Ch7 & & & ND & NS & 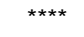 & **** & 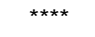 & 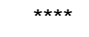 & $\star \star \star *$ & $* \star *$ & 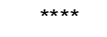 \\
\hline Ch11 & & & & ND & 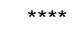 & **** & 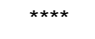 & 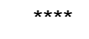 & ** & * & $\star * * * *$ \\
\hline Ch12 & & & & & ND & **** & 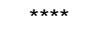 & 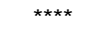 & $\star \star \star \star *$ & 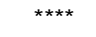 & 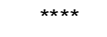 \\
\hline Ca386 & & & & & & ND & * & NS & 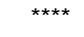 & 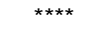 & 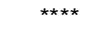 \\
\hline Ca432 & & & & & & & ND & NS & 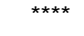 & **** & NS \\
\hline Ca446 & & & & & & & & ND & 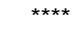 & 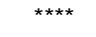 & * \\
\hline Ca881 & & & & & & & & & ND & NS & 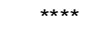 \\
\hline Ca885 & & & & & & & & & & ND & $\star * * \star *$ \\
\hline
\end{tabular}

ND, not determined. NS, not significant.

$\begin{array}{llll}\text { LIPCh1 } & \text { LIPCh3 } & \text { Ca881 } & \text { Ca386 } \\ \text { LIPCh6 } & \text { LIPCh7 } & \text { Ca885 } & \text { Ca432 } \\ \text { LIPCh12 } & \text { LIPCh11 } & & \text { Ca446 } \\ & & & \text { SC5314 }\end{array}$

Virulence

Figure 1. Survival curves of Galleria mellonella infected with $5 \times 10^{4}$ yeasts of (A) C. auris (Ca432; Ca386; Ca446; Ca881 and Ca885) or (B) C. haemulonii species complex (LIPCh1; LIPCh3; LIPCh6; LIPCh7; LIPCh11; LIPCh12). Survival was measured for 10 days. C. albicans SC5314 was used as control group; (C) statistical comparison of each survival curve and subsequently classification in 4 virulence groups. Data are representative of three independent experiments. Long-rank (Mantel-Cox) test, $p<0.05$. ${ }^{*} p<0.02,{ }^{* *} p<0.001,{ }^{* * *} p<0.0005$ and $p^{* * * *} p<0.0001$. 


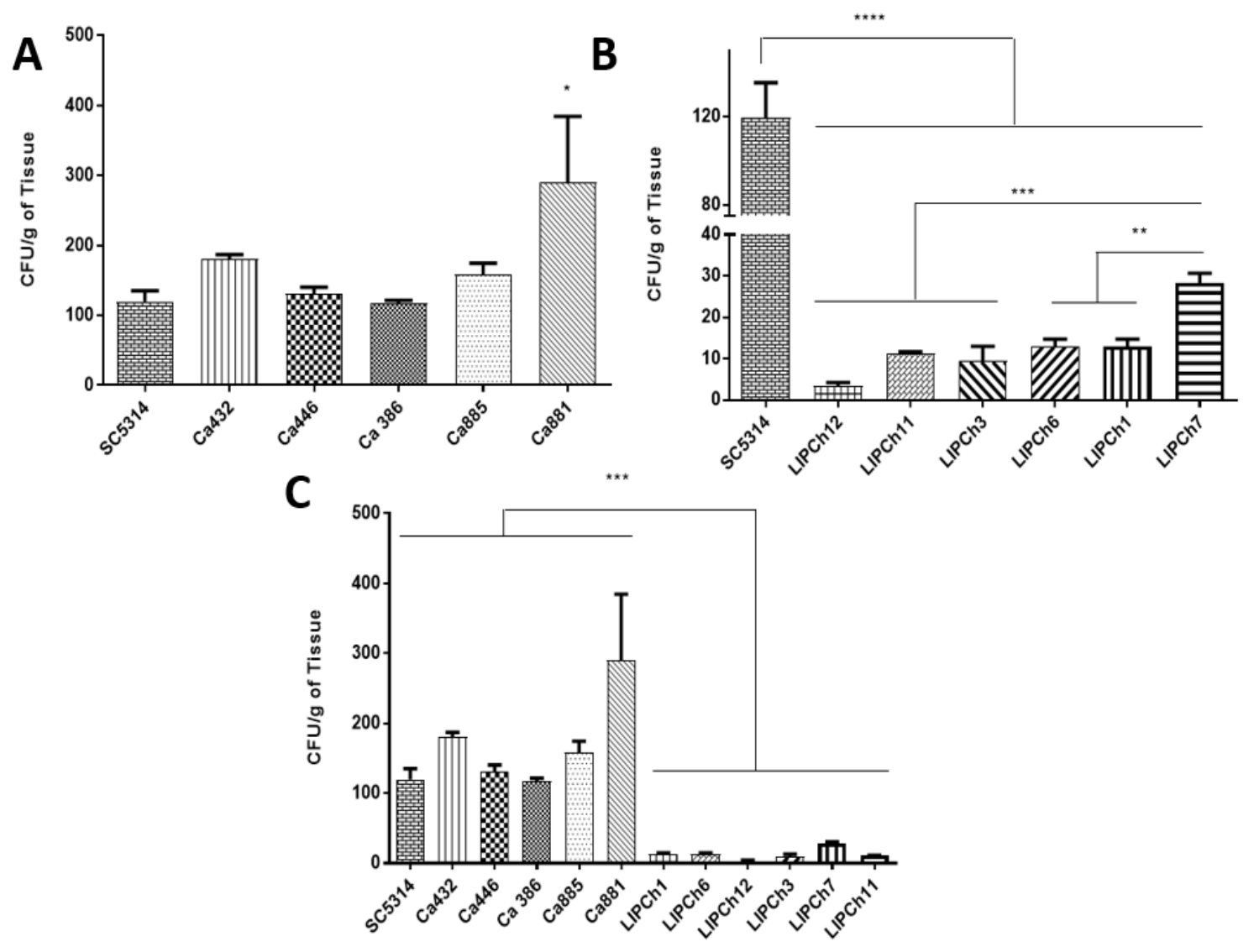

Figure 2. Galleria mellonella larvae were infected with $5 \times 10^{4}$ yeasts of C. auris (Ca432; Ca446; Ca386; Ca885; Ca881) or C. haemulonii complex (LIPCh1; LIPCh6; LIPCh12; LIPCh3; LIPCh7; LIPCh11) and sacrificed $3 \mathrm{~h}$ or 3 days after infection, respectively. The colony forming units (CFUs) were quantified after $24 \mathrm{~h}$ of growth in Sabouraud agar plates. (A) CFU of larvae infected with different strains of C. auris. (B) CFU of larvae infected with different strains of C. haemulonii complex. (C) Comparison among all species tested. As positive control C. albicans SC5314 was used. Data are representative of three independent experiments. ${ }^{*} p<0.05,{ }^{* *} p<0.001$ and ${ }^{* *} p<0.0001$. Error bars represent standard deviations between samples (biologic replicates) from the same group.

3.4. Virulence of C. auris and C. haemulonii in G. mellonella Model is Associated with Decrease in Phagocytosis

In the phagocytosis assay, we observed that G. mellonella larvae infected with the most virulent strains of $C$. auris (432 and 386) showed a significant decrease in hemocytes and remarkable cell lysis compared with larvae infected with $C$. haemulonii complex or those that received less virulent strains. Another important point is that larvae infected with the most virulent species of the $C$. haemulonii complex (LIPCh3, LIPCh7 and LIPCh11) presented a significant decrease in phagocytosis when compared with the less virulent strains of the same fungal species complex (LIPCh1, LIPCh6 and LIPCh12) (Figure 4). Larvae infected with the species of the C. haemulonii complex showed no significant decrease in hemocyte density and no cell lysis as observed in tests carried out with different strains of C. auris.

3.5. (C) auris and C. haemulonii Complex Infection have the Same Mortality Profile in an Immunosuppressed Animal Model

In the murine experimental model, observed for 30 consecutively days, no statistically significant difference in mortality was found between mice infected with C. auris (432 and 446) or with the species of the C. haemulonii complex (LIPCh3 and LIPCh11). However, up to the 30th day of infection, 
the mice mortality infected with C. auris (Ca446) was 100\%. Animals infected with C. auris (Ca432) presented a mortality similar $(80 \%)$ to those infected with the control strain of $C$. albicans ATCC10231. Female BALB/c mice that were inoculated with C. haemulonii (LIPCh3) and C. haemulonii var. vulnera (LIPCh11) had a survival close to 50\% (Figure 5).

\subsection{Biofilm Formation by C. auris and C. haemulonii Complex}

The virulent $C$. auris isolates Ca386 and Ca432 presented the greatest ability to generate biofilm compared to those less virulent as Ca881 and Ca885 (Figure 6A). In relation to the species of the C. haemulonii complex, LIPCh3 strain showed the highest metabolic activity in the biofilm formation when compared with the lowest virulent species LIPCh1 from C. duobushaemulonii. Similarly, the LIPCh11 strain of the C. haemulonii complex presented a low biofilm viability (Figure 6B). Visually, some differences in the biofilm appearance formed by the species of the $C$. haemulonii complex were observed. The biofilm produced by the $C$. haemulonii complex was thinner than those formed by C. auris. In addition, biofilm metabolic activity differences were observed in $C$. auris and the species of the $C$. haemulonii complex (see the $\mathrm{Y}$ axis in Figure 6A,B). In both experiments, C. albicans SC5314 was used as positive control due to its capacity to form a very highly heterogeneous biofilm.

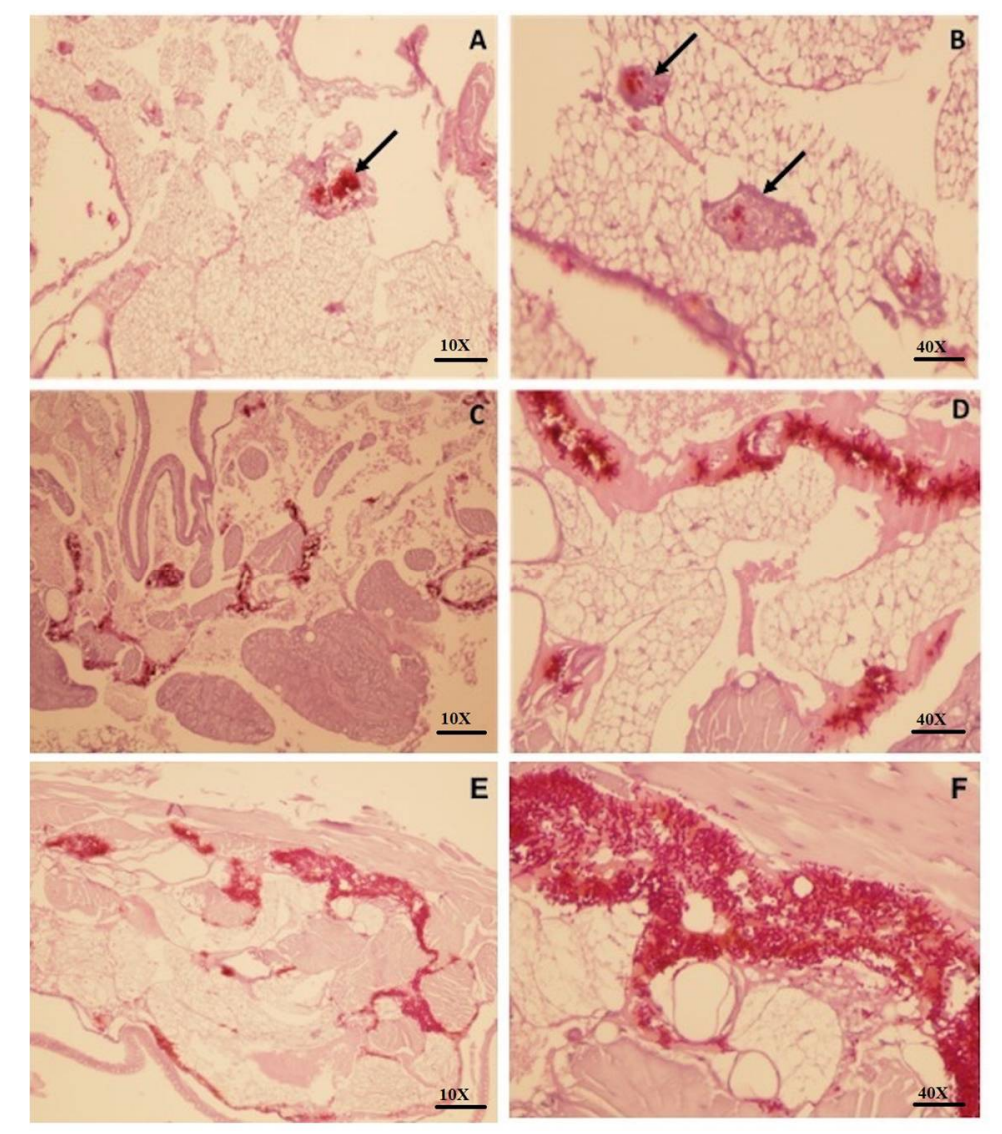

Figure 3. Galleria mellonella larvae were infected with $5 \times 10^{4}$ yeasts of $C$. auris and C. haemulonii complex and sacrificed $3 \mathrm{~h}$ or 3 days after infection, respectively. The tissue was preserved in formalin and then stained with periodic acid-Schiff (PAS). (A) Larvae infected with C. haemulonii complex 10x; (B) larvae infected with C. haemulonii complex 40x; (C) larvae infected with C. auris (432) 10x; (D) larvae infected with C. auris (432) 40×; (E) larvae infected with C. auris (386) 10×; (F) larvae infected with C. auris (386) 40×. Black arrows show granuloma-like cell aggregates in the tissue. 


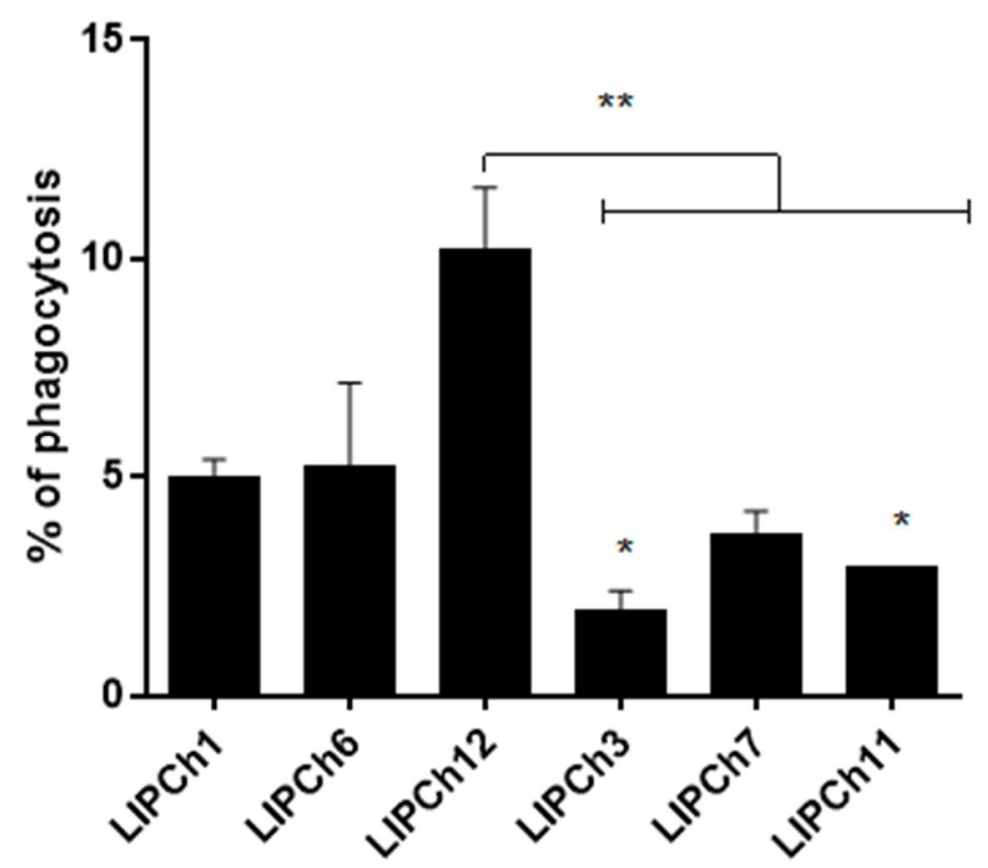

Figure 4. Larvae were infected with $5 \times 10^{4}$ cells of C. haemulonii complex (LIPCh1; LIPCh6; LIPCh12; LIPCh3; LIPCh7; LIPCh11) and after 3 days of infection the hemolymph was extracted. A smear in glass slide was prepared and stained with Wright dye. Per group were used 100 hemocytes to obtain the phagocytic percentage. ${ }^{* *}$ Indicates statistical significance ANOVA with post-Tukey's test, $p<0.05$ and * Indicates statistical significance $t$-test compared to C. haemulonii with low virulence (Ch1 and Ch6). Error bars represent standard deviations between samples (biologic replicates) from the same group.

\section{Survival curve of BALB/c mice}

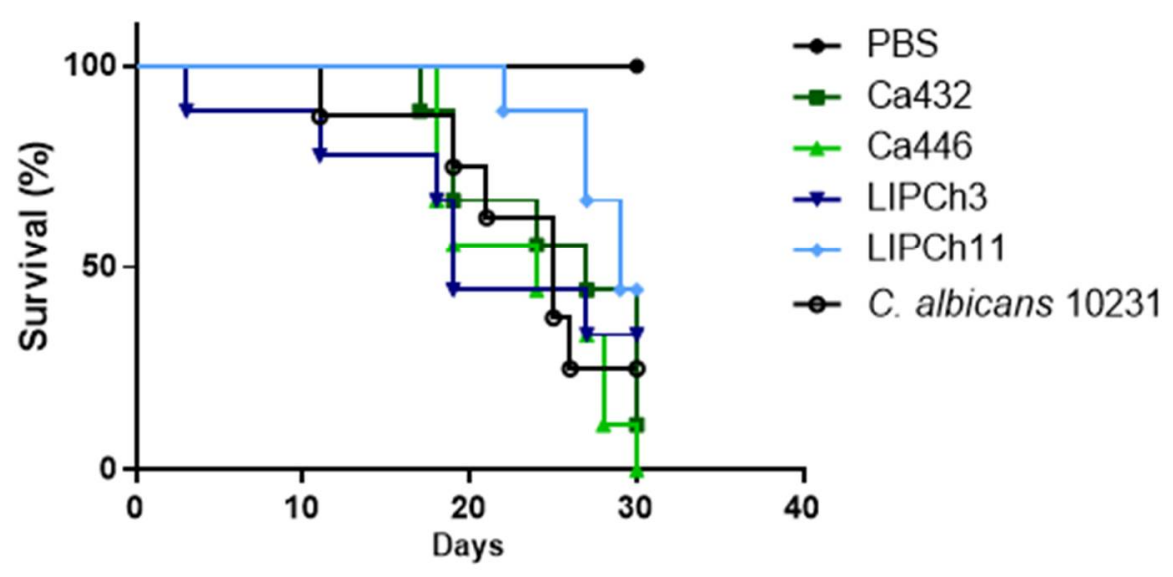

Figure 5. Animals ( $n=10$ /group) were immunosuppressed with cyclophosphamide and infected with $10^{4}$ yeasts of C. auris (Ca432 and Ca446), C. haemulonii complex (LIPCh3 and LIPCh11) or C. albicans 10,231 (control group). Animal survival was observed for 30 days after infection. Significance compared to PBS-inoculated group (Long-rank test, $p<0.05$ ). Data are representative of three independent experiments. 
A

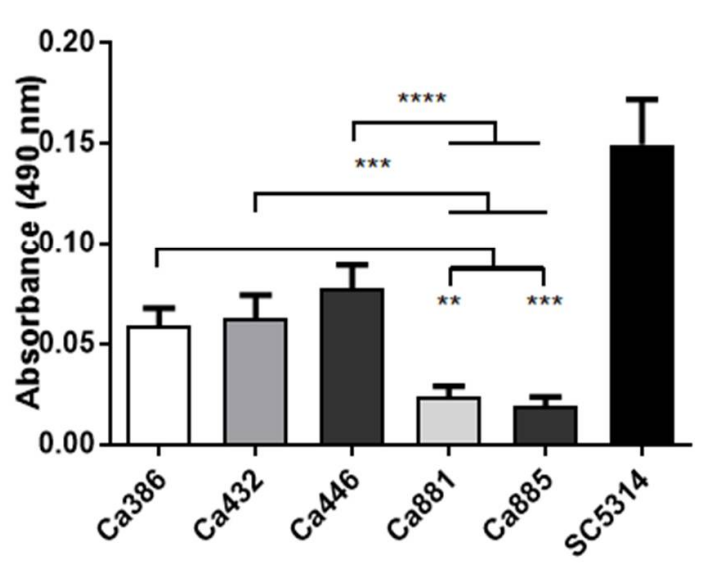

B Biofilm 24h C. haemulonii

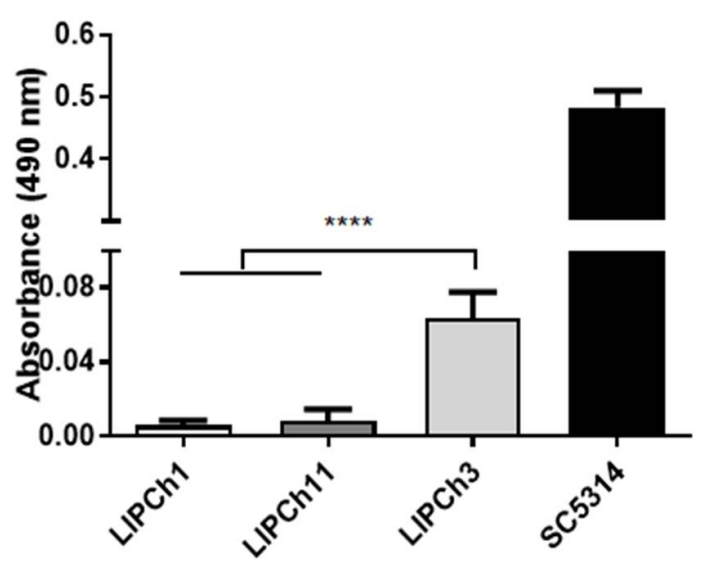

Figure 6. C. auris (A) and C. haemulonii (B) isolates were incubated in RPMI medium and after $24 \mathrm{~h}$, the biofilm formation was revelated by XTT/menadione addition. The colorimetric reaction was read spectrophotometrically $(490 \mathrm{~nm})$. Statistic differences were observed between the isolates of high and low virulence in both experiments. C. albicans SC5314 was used as positive control. ${ }^{* *} p<0.05,{ }^{* * *} p<0.001,{ }^{* * *} p<0.0001$. Error bars represent standard deviations between samples (biologic replicates) from the same group.

\section{Discussion}

In the present work, we evaluated the pathogenic capacity of cryptic species of the genus Candida using both in vitro and in vivo approaches. One of them is $C$. auris, recognized for its resistance and persistence on the surfaces and the other yeasts belong to the $C$. haemulonii complex (C. haemulonii, C. haemulonii var. vulnera and C. duobushaemulonii), which are phylogenetically related to C. auris.

As observed in this study, the survival of $C$. auris infected larvae was relatively short compared to larvae infected with the species of the $C$. haemulonii complex, suggesting that the $C$. auris strains utilized in this survey are more pathogenic than those from the $C$. haemulonii complex. Similar results were obtained by Gandra et al. (2020) [25], who described that C. haemulonii was less virulent than C. albicans in the G. mellonella model. Moreover, in that same work, it was described that to obtain comparable mortality rates between these two species, it was necessary to use an inoculum ten times larger of C. haemulonii yeasts [25]. On the other hand, the survival curve of G. mellonella larvae, infected with some strains of $C$. auris showed a high pathogenicity that was very similar to that observed in larvae infected with the $C$. albicans strain used as positive control. In the same way, Sherry and coworkers (2017), using the G. mellonella model, found that non-aggregating C. auris strains were more virulent than some C. albicans strains tested [26].

Regarding the fungal burden in the experimental model of G. mellonella, it is important to note that the growth of $C$. auris was very similar to the strain of $C$. albicans used as positive control; $C$ auris (Ca881) presented the major fungal burden of all C. auris strains and the positive control (SC5314). However, in the survival assay, the Ca881 strain was located within the second group of more virulent strains (Ca881 and Ca885) causing 100\% mortality on the sixth day of infection. Results of the fungal burden assay indicated that despite the high concentration of CFU produced by the Ca881 strain, other strains such as Ca386, Ca446 and Ca432 are more virulent and caused earlier mortality of infected larvae. A previous study, using strains of C. auris obtained in medical centers in the United Kingdom, indicated that some strains of this species may present a virulence significantly greater than others in terms of the kinetics of larval death and the number of dead larvae, mainly when $C$. auris does not form cellular aggregates [27]. Our results strongly suggest that the C. auris strains utilized are more virulent in the invertebrate model of G. mellonella than those from the C. haemulonii complex (Figure 1). 
Histopathology of G. mellonella larvae infected with C. auris and the species of the C. haemulonii complex showed a more aggressive infection process into the tissues of the larvae exposed to $C$. auris. Granuloma-like formation was a constant in the histopathology of larvae exposed to C. haemulonii complex strains (arrows Figure 3) probably due to the reduced evasion capacity of these yeasts against larvae immune responses corroborated by a reduced fungal burden and the larvae survival prolongation. As we noted at the moment to grow the strains, C. auris duplicated after two hours, while yeasts of the $C$. haemulonii complex duplicated after three hours. Probably, the growth rate also is related to the virulence profile exhibit for the yeasts analyzed. An interesting feature is that the formation of pseudohyphae was not observed in all the Candida species tested herein. In the case of C. auris, some authors highlight the inability of this species to produce pseudohyphae, germ tube, chlamydoconidia and chlamydospores [28]. On the other hand, different authors have described that the formation of a rudimentary and occasional pseudohyphae may occur, specific to some strains or under certain conditions, as stress response [29]. The formation of hyphae, pseudohyphae and germ tube in other yeast species such as C. albicans and C. tropicalis has been associated with high virulence [30]. In the near future, it will be important to deeply analyze the virulence factors of C. auris that could avoid the larvae immune system and that would be absent in the $C$. haemulonii complex strains.

The phagocytosis plays an important role in the innate immune response of G. mellonella. Hemocytes represent the main element of the cellular response of these larvae. In our study, we observed a drastic decrease in the number of hemocytes of larvae infected with $C$. auris due to a generalized lysis that does not allow us to carry out the phagocytosis rate analysis for all C. auris strains tested. This lysis profile of hemocytes observed in the case of $C$. auris is probably related to the possible virulence factors produced by this fungus that destroy the larval hemocytes. In the same way, we suggest that $C$. haemulonii species complex also have virulence factors that protect it from being totally phagocyted, but there are not as efficient as those of $C$. auris. The hemocyte lysis observed also can be related to the high and rapid death rates of the larvae with only two days post-infection that we noticed in the survival test. Previous works had shown that there is a close relationship between the number of hemocytes and the larvae survival rate, where larvae inoculated with high pathogenic strains have a reduced density of hemocytes, while those inoculated with low pathogenic strains show only a small variation in their hemocytes density [31,32]. In the case of larvae infected with the species of the $C$. haemulonii complex, was possible to carry out the experiment. We observed the lowest percentage of phagocytosis in the larvae infected with the most virulent strains of the C. haemulonii complex as LIPCh3, LIPCh7 and C. haemulonii var. vulnera (LIPCh11), probably due to the hemocyte decrease or to the production of virulence factors such as hydrolytic enzymes etc. However, it is important to note that phagocytosis can already be observed within a few hours after infection with this Candida species as referred by Silva et al. 2018 [33].

To study the disseminated candidiasis model, we used immunosuppressed BALB/c mice with the aim of better understand the pathogenicity processes of the different Candida species analyzed. Why we use immunosuppressed mice is that it allows us to better observe the differences in pathogenicity of the yeasts, since mice with a normal immune system could resolve the infection on their own or it would be necessary to use a very concentrated inoculum to observe differences in the infection process. We utilized the intravenous challenge model of disseminated infection that is commonly used to determine the virulence of $C$. albicans strains [34]. When we observed the virulence of $C$. auris and the species of the $C$. haemulonii complex in the murine experimental model, there was no significant difference in the survival of the mice infected with the yeasts tested. Some authors have described the poor ability of $C$. auris to infect and spread in mice compared with other Candida species [35]. However, Ben-Ami et al. (2017) [30] suggested a high virulence and pathogenicity of C. auris in mice, but specifically using strains that form cellular aggregates. Other studies highlighted the virulence of $C$. auris compared to other species such as C. albicans and C. glabrata in the immunocompetent murine model [36]. After all, we believe it is necessary to carry out more specific studies in more 
experimental models that could help us to better understand the process of infection and colonization of Candida auris that becomes more frequent and resistant.

Another important characteristic in the pathogenicity of Candida is the ability to form biofilm, which protects the yeasts from the action of different cells of the immune system-as well as the action of antifungals. When we analyzed the capacity of biofilm formation of the two species here studied by the XTT assay, we suggest that $C$. auris has a greater capacity to form viable biofilm than the species of the $C$. haemulonii complex. However, more specific experiments as the crystal violet test could be done in a near future to strengthen these results. Unexpectedly, the species C. haemulonii var. vulnera LIPCh11 did not exhibit the same biofilm behavior as LIPCh3 despite being also a highly virulent species in relation with the fungal burden and the larvae mortality. Our results corroborate what was found and described by Sherry and collaborators in 2017 [26]. However, it is important to note that these two species of Candida presented a lower biofilm production compared with the positive control (C. albicans SC5314). Hence, that, in the case of the strains of $C$. auris and C. haemulonii complex herein analyzed, the biofilm formation seems not to be a remarkable pathogenic characteristic.

In summary, the strains of $C$. auris present a significantly higher virulence compared to the species belonging to the $C$. haemulonii complex used in the experimental model of G. mellonella. However, in the murine model the difference in virulence of these fungal species was not as marked. However, the evasion of phagocytosis, as well as the biofilm formation of the C. auris strains, undoubtedly present important virulence factors that make this yeast an agent more adapted to the infection process in the host.

Author Contributions: J.E.M. designed, performed and supervised experiments, analyzed data and wrote the manuscript. L.M.R., L.d.S.D., L.A.R. and L.S.R., designed and performed experiments and analyzed data. A.L.S.S., C.P.T., designed and supervised experiments, analyzed data and revised the manuscript. C.M.P.-G. conceived the study, participated in its design and coordination the manuscript. All authors have read and agreed to the published version of the manuscript.

Funding: This work was supported by Fundação de Amparo à Pesquisa do Estado de São Paulo (FAPESP), Fundação de Amparo à Pesquisa do Estado do Rio de Janeiro (FAPERJ), Conselho Nacional de Desenvolvimento Científico e Tecnológico (CNPq), Coordenação de Aperfeiçoamento de Pessoal de Nível Superior-Brasil-CAPES (http://www.capes.gov.br/), School of Medicine and Health Sciences, Universidad del Rosario, Vicerrectoría de Investigación de la Pontificia Universidad Javeriana and (MINCIENCIAS) through contract RC-715-2018.

Acknowledgments: We are grateful to the technical support and collaborators of the Laboratorio de Fungos Dimórficos Patogênicos from the University of São Paulo, Brazil and to Soraya E. Morales-López of the Popular University of Cesar, Colombia for giving us the strains of Candida auris utilized in this study.

Conflicts of Interest: The authors report no conflicts of interest. The authors only are responsible for the content and the writing of the study.

\section{References}

1. Lockhart, S.R.; Etienne, K.A.; Vallabhaneni, S.; Farooqi, J.; Chowdhary, A.; Govender, N.P.; Colombo, A.L.; Calvo, B.; Cuomo, C.A.; Desjardins, C.A.; et al. Simultaneous emergence of multidrug-resistant candida auris on 3 continents confirmed by whole-genome sequencing and epidemiological analyses. Clin. Infect. Dis. 2017, 64, 134-140. [CrossRef] [PubMed]

2. Kim, M.; Shin, J.H.; Sung, H.; Lee, K.; Kim, E.; Ryoo, N.; Lee, J.-S.; Jung, S.-I.; Park, K.H.; Kee, S.J.; et al. Candida haemulonii and closely related species at 5 university hospitals in Korea: Identification, antifungal susceptibility, and clinical features. Clin. Infect. Dis. 2009, 48, e57-e61. [CrossRef]

3. Satoh, K.; Makimura, K.; Hasumi, Y.; Nishiyama, Y.; Uchida, K.; Yamaguchi, H. Candida auris sp. nov., a novel ascomycetous yeast isolated from the external ear canal of an inpatient in a Japanese hospital. Microbiol. Immunol. 2009, 53, 41-44. [CrossRef] [PubMed]

4. Chowdhary, A.; Sharma, C.; Duggal, S.; Agarwal, K.; Prakash, A.; Singh, P.K.; Jain, S.; Kathuria, S.; Randhawa, H.S.; Hagen, F.; et al. New clonal strain of Candida auris, Delhi, India. Emerg. Infect. Dis. 2013, 19, 1670-1673. [CrossRef] [PubMed]

5. Magobo, R.E.; Corcoran, C.; Seetharam, S.; Govender, N.P. Candida auris-Associated candidemia, South Africa. Emerg. Infect. Dis. 2014, 20, 1250-1251. [CrossRef] 
6. Emara, M.; Ahmad, S.; Khan, Z.; Joseph, L.; Al-Obaid, I.M.; Purohit, P.; Bafna, R. Candida auris Candidemia in Kuwait, 2014. Emerg. Infect. Dis. 2015, 21, 1091-1092. [CrossRef] [PubMed]

7. Nucci, M.; Queiroz-telles, F.; Alvarado-matute, T.; Tiraboschi, I.N.; Cortes, J.; Zurita, J.; Guzman-Blanco, M.; Santolaya, M.E.; Thompson, L.; Sifuentes-Osornio, J.; et al. Epidemiology of Candidemia in Latin America: A laboratory-based survey. PLoS ONE 2013, 8, e59373. [CrossRef] [PubMed]

8. Morales-López, S.E.; Parra-Giraldo, C.M.; Ceballos-Garzón, A.; Martínez, H.P.; Rodríguez, G.J.; Álvarez-Moreno, C.A.; Rodríguez, J.Y. Invasive infections with multidrug-resistant yeast Candida auris, Colombia. Emerg. Infect. Dis. 2017, 23, 162-164. [CrossRef]

9. Parra-Giraldo, C.M.; Valderrama, S.L.; Cortes-Fraile, G.; Garzón, J.R.; Ariza, B.E.; Morio, F.; Linares-Linares, M.Y.; Ceballos-Garzón, A.; de la Hoz, A.; Hernandez, C.; et al. First report of sporadic cases of Candida auris in Colombia. Int. J. Infect. Dis. 2018, 69, 63-67. [CrossRef]

10. Escandón, P.; Chow, N.A.; Caceres, D.H.; Gade, L.; Berkow, E.L.; Armstrong, P.; Rivera, S.; Misas, E.; Duarte, C.; Moulton-Meissner, H.; et al. Molecular epidemiology of Candida auris in Colombia reveals a highly related, countrywide colonization with regional patterns in amphotericin b resistance. Clin. Infect. Dis. 2019, 68, 15-21. [CrossRef]

11. Pappas, P.G.; Lionakis, M.S.; Arendrup, M.C.; Ostrosky-Zeichner, L.; Kullberg, B.J. Invasive candidiasis. Nat Rev Dis Prim. 2018, 4, 18026. [CrossRef]

12. Vallabhaneni, S.; Kallen, A.; Tsay, S.; Chow, N.; Welsh, R.; Kerins, J. Investigation of the first seven reported cases of Candida auris, a globally emerging invasive, multidrug-resistant fungus-United States, May 2013August 2016. Am. J. Transpl. 2017, 17, 296-299. [CrossRef] [PubMed]

13. Schelenz, S.; Hagen, F.; Rhodes, J.L.; Abdolrasouli, A.; Chowdhary, A.; Hall, A.; Ryan, L.; Shackleton, J.; Trimlett, R.; Meis, J.F.; et al. First hospital outbreak of the globally emerging Candida auris in a European hospital. Antimicrob. Resist. Infect. Control. 2016, 5, 1-7. [CrossRef]

14. Ramos, L.S.; Figueiredo-Carvalho, M.H.G.; Barbedo, L.S.; Ziccardi, M.; Chaves, A.L.S.; Zancopé-Oliveira, R.M.; Pinto, M.R.; Sgarbi, D.B.G.; Dornelas-Ribeiro, M.; Branquinha, M.H.; et al. Candida haemulonii complex: Species identification and antifungal susceptibility profiles of clinical isolates from Brazil. J. Antimicrob. Chemother. 2015, 70, 111-115. [CrossRef]

15. Shackleton, J.; Schelenz, S.; Rochon, M.; Hall, A.; Ryan, L.; Cervera-Jackson, R. The impact of environmental decontamination in a Candida auris outbreak. J. Hosp. Infect. 2016, 94, S24-S134. [CrossRef]

16. Sarma, S.; Kumar, N.; Sharma, S.; Govil, D.; Ali, T.; Mehta, Y.; Rattan, A. Candidemia caused by amphotericin B and Fluconazole resistant Candida auris. Indian J. Med. Microbiol. 2013, 31, 90. [CrossRef]

17. Rossato, L.; Colombo, A.L. Candida auris: What have we learned about its mechanisms of pathogenicity? Front Microbiol. 2018, 9, 1-6. [CrossRef]

18. Lavarde, V. Peritonite mycosique a Torulopsis haemulonii. Bul. Soc. Fr. Mycol. Med. 1984, 13, 173-176.

19. Khan, Z.U.; Al-Sweih, N.A.; Ahmad, S.; Al-Kazemi, N.; Khan, S.; Joseph, L.; Chandy, R. Outbreak of fungemia among neonates caused by Candida haemulonii resistant to amphotericin B, itraconazole, and fluconazole. J. Clin. Microbiol. 2007, 45, 2025-2027. [CrossRef]

20. Muro, M.D.; Motta, F.D.A.; Burger, M.; Melo, A.S.D.A.; Dalla-Costa, L.M. Echinocandin resistance in two Candida haemulonii isolates from pediatric patients. J. Clin. Microbiol. 2012, 50, 3783-3785. [CrossRef]

21. Ruan, S.; Kuo, Y.; Huang, C.; Hsiue, H.; Hsueh, P. Infections due to Candida haemulonii: Species identification, antifungal susceptibility and outcomes. Int. J. Antimicrob. Agents. 2010, 35, 85-88. [CrossRef]

22. Cendejas-Bueno, E.; Kolecka, A.; Alastruey-Izquierdo, A.; Theelen, B.; Groenewald, M.; Kostrzewa, M.; Boekhout, T. Reclassification of the Candida haemulonii complex as Candida. J. Clin. Microbiol. 2012, 50, 3641-3651. [CrossRef] [PubMed]

23. Rossi, D.C.; Muñoz, J.E.; Carvalho, D.D.; Belmonte, R.; Faintuch, B.; Borelli, P.; Daffre, S. Therapeutic use of a cationic antimicrobial peptide from the spider Acanthoscurria gomesiana in the control of experimental candidiasis. BMC Microbiol. 2012, 12, 28. [CrossRef]

24. Pierce, C.G.; Uppuluri, P.; Tristan, A.R.; Jr, F.L.W.; Mowat, E.; Ramage, G.; Lopez-Ribot, J.L. A simple and reproducible 96 well plate-based method for the formation of fungal biofilms and its application to antifungal susceptibility testing. Nat. Protoc. 2008, 3, 1494-1500. [CrossRef] [PubMed]

25. Gandra, R.M.; McCarron, P.; Viganor, L.; Fernandes, M.F.; Kavanagh, K.; McCann, M.; Devereux, M. In vivo activity of Copper(II), manganese(II), and silver(I) 1,10-phenanthroline chelates against Candida haemulonii using the Galleria mellonella model. Front Microbiol. 2020, 11, 1-15. [CrossRef] 
26. Sherry, L.; Ramage, G.; Kean, R.; Borman, A.; Johnson, E.M.; Richardson, M.D. Biofilm-forming capability of highly virulent, multidrug-resistant Candida auris. Emerg. Infect. Dis. 2017, 23, 328-331. [CrossRef] [PubMed]

27. Borman, A.M.; Szekely, A.; Johnson, E.M. Comparative pathogenicity of United Kingdom isolates of the emerging. mSphere 2016, 1, e00189-16. [CrossRef] [PubMed]

28. Azar, M.M.; Turbett, S.E.; Fishman, J.A.; Pierce, V.M. Donor-derived transmission of Candida auris during lung transplantation. Clin. Infect. Dis. 2017, 65, 1040-1042. [CrossRef]

29. Bravo Ruiz, G.; Ross, Z.K.; Gow, N.A.R.; Lorenz, A. Pseudohyphal growth of the emerging pathogen Candida auris is triggered by genotoxic stress through the $S$ Phase Checkpoint. mSphere 2020, 5, 1-17. [CrossRef]

30. Ben-Ami, R.; Berman, J.; Novikov, A.; Bash, E.; Shachor-Meyouhas, Y.; Zakin, S.; Finn, T. Multidrug resistant Candida haemulonii and Candida auris Tel Aviv, Israel. Emerg. Infect. Dis. 2017, 23, 195-203. [CrossRef]

31. Bergin, D.; Brennan, M.; Kavanagh, K. Fluctuations in haemocyte density and microbial load may be used as indicators of fungal pathogenicity in larvae of Galleria mellonella. Microbes Infect. 2003, 5, 1389-1395. [CrossRef] [PubMed]

32. Gago, S.; García-Rodas, R.; Cuesta, I.; Mellado, E.; Alastruey-Izquierdo, A. Candida parapsilosis, Candida orthopsilosis, and Candida metapsilosis virulence in the non-conventional host Galleria mellonella. Virulence 2014, 5, 278-285. [CrossRef] [PubMed]

33. Silva, L.N.; Campos-Silva, R.; Ramos, L.S.; Trentin, D.S.; Macedo, A.J.; Branquinha, M.H.; Santos, A.L. Virulence of Candida haemulonii complex in Galleria mellonella and efficacy of classical antifungal drugs: A comparative study with other clinically relevant non-albicans Candida species. FEMS Yeast Res. 2018, 18. [CrossRef] [PubMed]

34. Conti, H.R.; Huppler, A.R.; Whibley, N.; Gaffen, S.L. Animal models for Candidiasis. Curr. Protoc. Immunol. 2014. [CrossRef]

35. Larkin, E.; Hager, C.; Chandra, J.; Mukherjee, P.K. The emerging pathogen Candida auris: Growth phenotype, virulence factors, activity of antifungals, and effect of SCY-078, a novel glucan synthesis inhibitor, on growth morphology and biofilm formation emily. Antimicrob. Agents Chemother. 2017, 61, e02396-16. [CrossRef] [PubMed]

36. Fakhim, H.; Vaezi, A.; Dannaoui, E.; Chowdhary, A.; Nasiry, D.; Faeli, L.; Meis, J.F.; Badali, H. Comparative virulence of Candida auris with Candida haemulonii, Candida glabrata and Candida albicans in a murine model. Mycoses 2018, 61, 377-382. [CrossRef] 